\title{
A Three-Dimensional Molecular Perovskite Ferroelastic with Bi-Step Switching of Quadratic Nonlinear Optical (NLO) Properties Tuned by Molecular Chiral Design
}

Zhi-Bo Liu, ${ }^{\S}$ Lei He, ${ }^{\S}$ Ping-Ping Shi, Qiong Ye*, Da-Wei Fu*

Jiangsu Key Laboratory for Science and Applications of Molecular Ferroelectrics,

Southeast University, Nanjing 211189, People's Republic of China 


\section{Supporting Information}

Synthesis. All reagents were obtained from commercial sources and used without further purification. (2-Hydroxy-propyl)-tripropyl-ammonium and (2-Hydroxy-1methyl-ethyl)-tripropyl-ammonium cations were synthesized by exhaustive alkylation of the corresponding amine with 1-iodopropane.

Synthesis of $1 . \mathrm{K}_{2} \mathrm{CO}_{3}(42 \mathrm{~g}, 0.3 \mathrm{M})$ was added into a brown three-necked flask equipped with a reflux condenser. Anhydrous acetonitrile $(100 \mathrm{~mL})$ was added into reaction flask under nitrogen gas at room temperature. Then, 1-Amino-2-propanol (9 $\mathrm{mL}, 0.12 \mathrm{M}$ ) was added to the solvent using a syringe and stirred for 10 minutes. While stirring, 1-iodopropane ( $40 \mathrm{~mL}, 0.41 \mathrm{M})$ was slowly added to the mixed solution. Then, the reaction solution was refluxed for $48 \mathrm{~h}$ in dark. After $48 \mathrm{~h}$, the mixture was cooled to room temperature and then filtrated to remove solid (mainly $\mathrm{K}_{2} \mathrm{CO}_{3}$ ). About a half of filtrate was evaporated by a rotary evaporator, yielding liquid with precipitates. The precipitates (mainly KI) were then removed by suction filtration. The remaining filtrate was further evaporated in vacuum, yielding white precipitates. The obtained white solid was purified by using ethyl acetate and ethanol for recrystallization purification in a refrigerator. The white solid is (2-hydroxy-propyl)-tripropyl-ammonium iodide. The chloride salt is obtained by substituting iodide using $\mathrm{Ag}_{2} \mathrm{CO}_{3}$ and hydrochloric acid in stoichiometric ratio. Electrospray cation mass spectrometry confirmed the purity of the ammonium cation (Figure S2a). The cubic colorless crystals were obtained by slow evaporation of aqueous solution containing $\mathrm{Na}(\mathrm{dca})(1.335 \mathrm{~g}, 15 \mathrm{mmol})$, $\mathrm{Mn}\left(\mathrm{NO}_{3}\right)_{2} \cdot 4 \mathrm{H}_{2} \mathrm{O}(1.7895 \mathrm{~g}, 5 \mathrm{mmol})$ and (2-hydroxy-propyl)-tripropyl-ammonium chloride (1.189 g, $5 \mathrm{mmol})$ at room temperature. After being placed for ten months, they are still clear and colorless crystals. They are relatively stable.

Synthesis of 2. $\mathrm{K}_{2} \mathrm{CO}_{3}(42 \mathrm{~g}, 0.3 \mathrm{M})$ was added into a brown three-necked flask equipped with a reflux condenser. Anhydrous acetonitrile $(100 \mathrm{~mL})$ was added into reaction flask under nitrogen gas at room temperature. Then, 2-Amino-1-propanol (9 $\mathrm{mL}, 0.11 \mathrm{M}$ ) was added to the solvent using a syringe and stirred for 10 minutes. While 
stirring, 1-iodopropane ( $40 \mathrm{~mL}, 0.41 \mathrm{M}$ ) was slowly added to the mixed solution. Then, the reaction solution was slowly heated to $85^{\circ} \mathrm{C}$ and refluxed for $48 \mathrm{~h}$ (kept in dark). After $48 \mathrm{~h}$, the mixture was cooled to room temperature and then filtrated to remove solid (mainly $\mathrm{K}_{2} \mathrm{CO}_{3}$ ). About a half of filtrate was evaporated by a rotary evaporator, yielding liquid with precipitates. The precipitates (mainly KI) were then removed by suction filtration. The remaining filtrate was further evaporated in vacuum, yielding yellow liquid with white precipitates. Ethyl acetate was added to the solid-liquid mixture and then filtered to obtain a white solid. The white solid was further washed with ethyl acetate and dried under atmospheric condition. The white solid is (2hydroxy-1-methyl-ethyl)-tripropyl-ammonium iodide. The chloride salt is obtained by substituting iodide using $\mathrm{Ag}_{2} \mathrm{CO}_{3}$ and hydrochloric acid in stoichiometric ratio. Electrospray cation mass spectrometry confirmed the purity of the ammonium cation (Figure S2b). The cubic colorless crystals were obtained by slow evaporation of aqueous solution containing $\mathrm{Na}(\mathrm{dca})(1.335 \mathrm{~g}, 15 \mathrm{mmol}), \mathrm{Mn}\left(\mathrm{NO}_{3}\right)_{2} \cdot 4 \mathrm{H}_{2} \mathrm{O}(1.7895 \mathrm{~g}$, $5 \mathrm{mmol})$ and (2-hydroxy-1-methyl-ethyl)-tripropyl-ammonium chloride (1.189 g, 5 mmol) at room temperature. After being placed for ten months, they are still clear and colorless crystals. They are relatively stable.

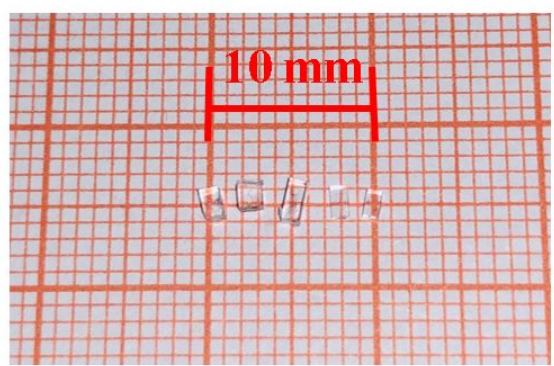

(a)

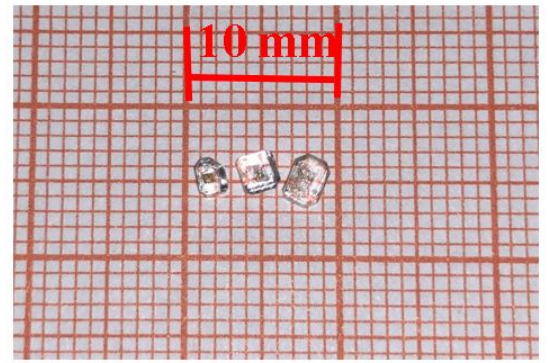

(b)

Figure S1. The crystal photos of $\mathbf{1}$ (a) and $\mathbf{2}$ (b). 


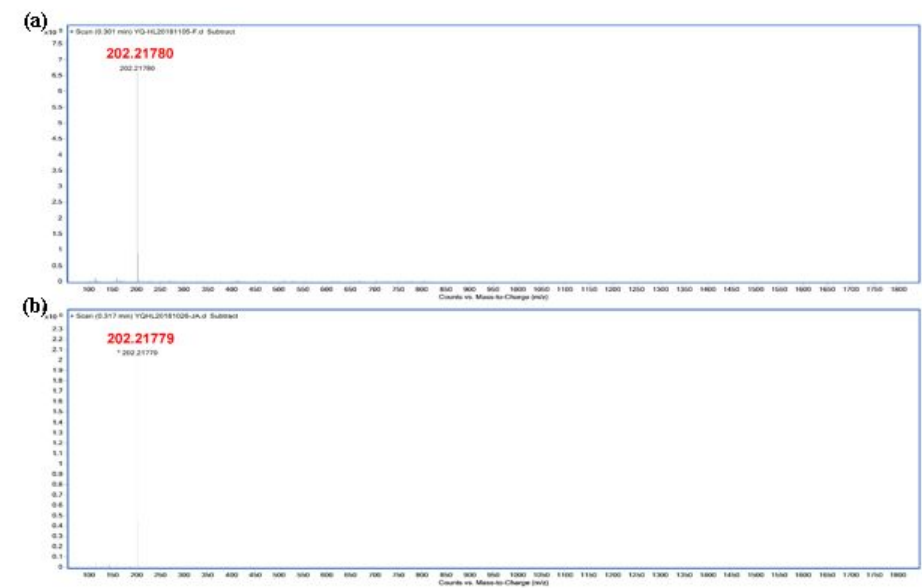

Figure S2. The electrospray cation mass spectrums of 1 (a) and 2 (b).

The formation of compounds $\mathbf{1}$ and $\mathbf{2}$ was verified by the PXRD patterns (Figure S3) and IR-60 spectrum (Figure S4). The spectra of the two compounds are basically the same, owing to the same functional groups. The four weak absorption peaks near 3000 $\mathrm{cm}^{-1}$ are $\mathrm{C}-\mathrm{H}$ stretching vibration of methyl and methylene groups. The peaks around 3400 and $2295 \mathrm{~cm}^{-1}$ are the characteristic $-\mathrm{OH}$ and $\mathrm{C} \equiv \mathrm{N}$ vibrations, respectively.
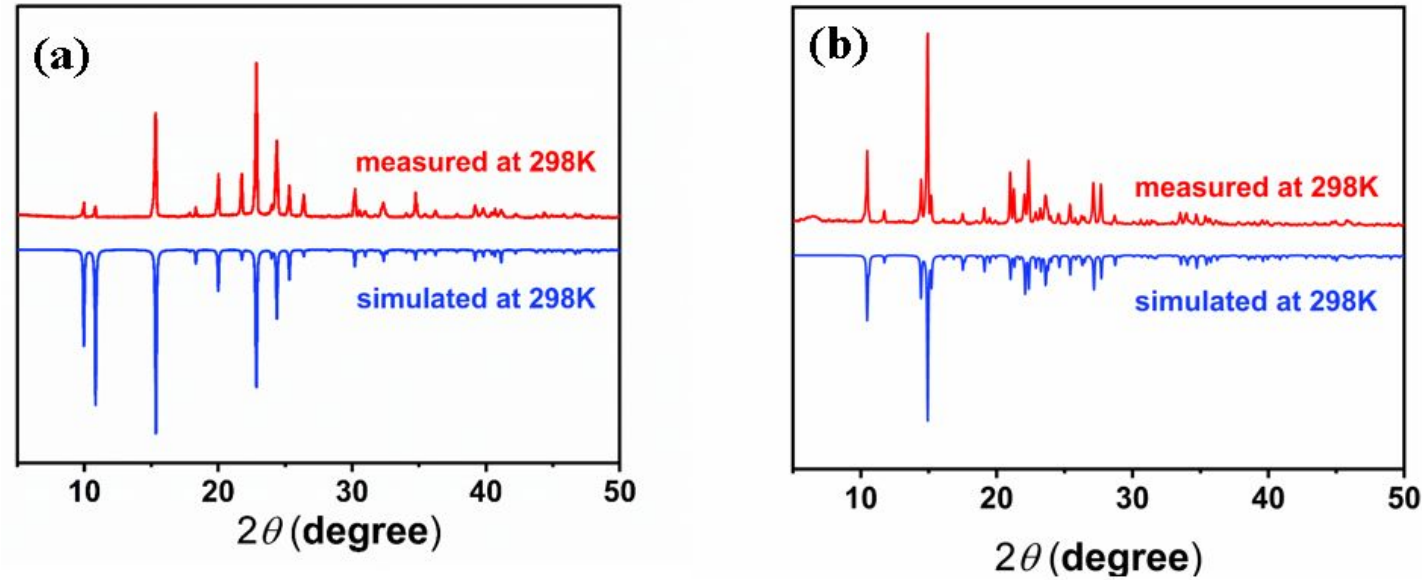

Figure S3. Powder XRD patterns of 1 (a) and 2 (b) at 298 K. 


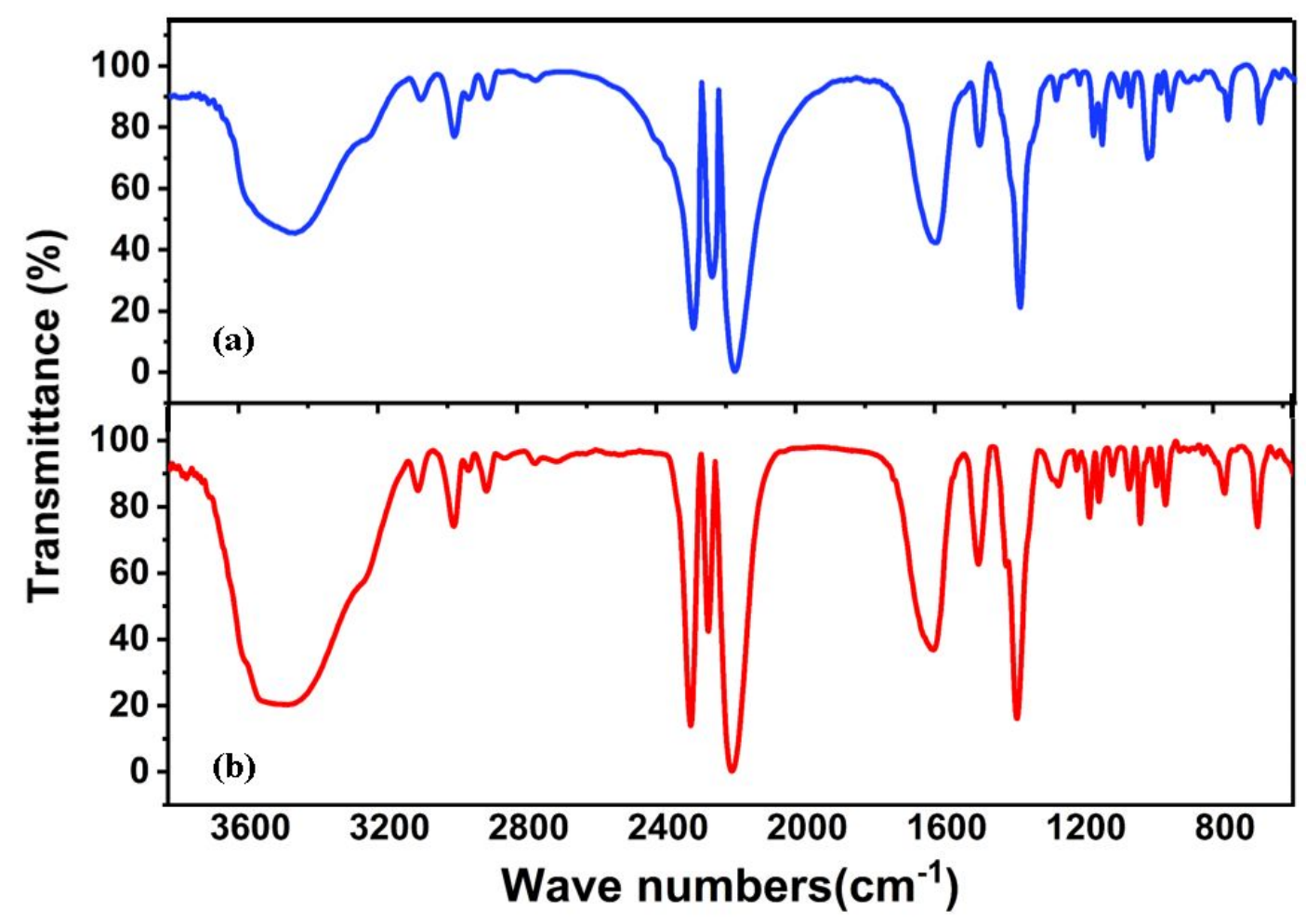

Figure S4. IR spectrums of $\mathbf{1}$ (a) and $\mathbf{2}$ (b) on a $\mathrm{KBr}$-diluted pellet at room temperature.

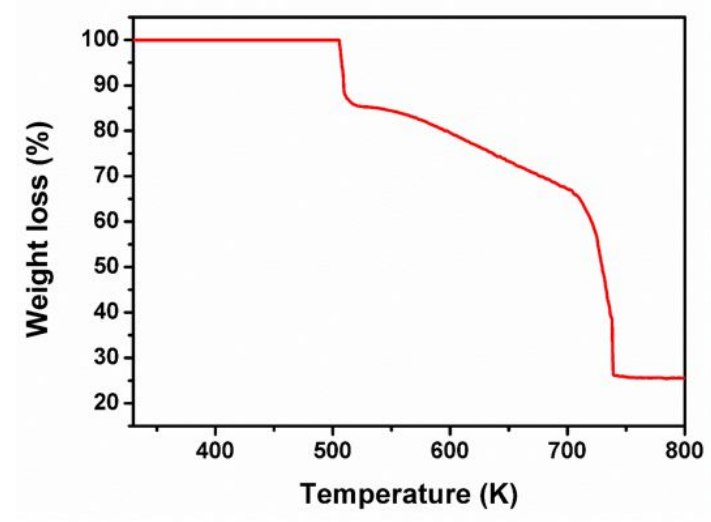

(a)

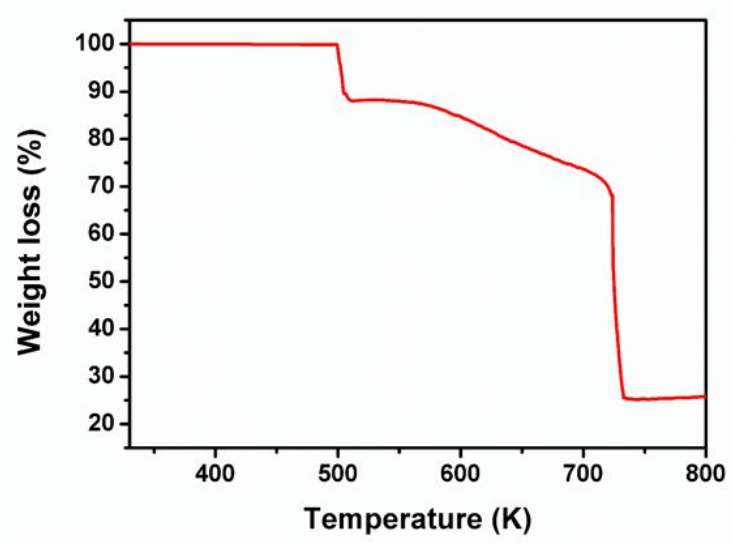

(b)

Figure S5. The TGA curves of 1 (a) and 2 (b).

The result indicates that the samples of $\mathbf{1}$ and $\mathbf{2}$ begin to decompose at about $506 \mathrm{~K}$ and $499 \mathrm{~K}$, respectively.

\section{Single Crystal X-ray Crystallography}

The single-crystal X-ray diffraction data of $\mathbf{1}$ and $\mathbf{2}$ were collected on a Rigaku Satum 724 diffractometer with Mo K $\alpha$ radiation $(\lambda=0.71073 \AA)$ at different temperature. The Crystal-clear software package (Rigaku, 2005) was used to perform data reduction and empirical absorption correction. Direct methods were used to solve the crystal structure, and the SHELXLTL software package (SHELX-97) was used to refine by full-matrix 
least-squares refinements based on $\mathrm{F}^{2}$. Non-H atoms were refined anisotropically and the $\mathrm{H}$ atoms were generated geometrically. The structure of packing views was drawn with DIAMOND (Brandenburg and Putz, 2005). Crystal data and structure refinement details of $\mathbf{1}$ and $\mathbf{2}$ are given in Table $\mathbf{S 1}$ and $\mathbf{S 2}$.

\section{Powder X-ray Diffraction}

A PANalytical X'Pert PRO X-ray diffractometer was used to measure 1 at $298 \mathrm{~K}$ and 2 at $293 \mathrm{~K} / 353 \mathrm{~K}$ by X-ray powder diffraction (PXRD). Diffraction patterns were collected in the $2 \theta$ range of $5-50^{\circ}$ with a step size of $0.02^{\circ}$.

\section{Thermal Measurements}

DSC experiments were carried out on a NETZSCH DSC200F3 instrument by heating and cooling the crystalline samples of 1 and 2 with a rate of $10 \mathrm{~K} / \mathrm{min}$ under a nitrogen atmosphere in aluminum crucibles.

\section{SHG Switching Measurements}

SHG switching experiment was measured with powder samples by pulsed Nd: YAG (1064 nm, Vibrant 355 II, OPOTEK).

\section{Ferroelastic Measurements}

For ferroelastic properties, ferroelastic domain observations were carried on an Olympus BX51TRF polarizing microscope. The temperature was controlled by the INSTEC HCC602 cooling / heating stage. Aqueous saturated solution $(20 \mu \mathrm{L})$ of 2 was dropped on a prepared indium tin oxide (ITO)-coated glass, and thin film was obtained at heating stage of $313 \mathrm{~K}$. According to a heating and cooling cycle, the transforming of domain structures of the thin film can be observed.

\section{Dielectric Constant Measurements}

Crystalline powdered samples of $\mathbf{1}$ and $\mathbf{2}$ deposited with carbon conductive glue painted on both sides were used to measure dielectric constant. The samples of $\mathbf{1}$ and $\mathbf{2}$ temperature-dependent dielectric constants were performed on a TongHui 2828 impedance analyzer at frequencies of $1 \mathrm{MHz}$ of $1 \mathrm{~V}$. 


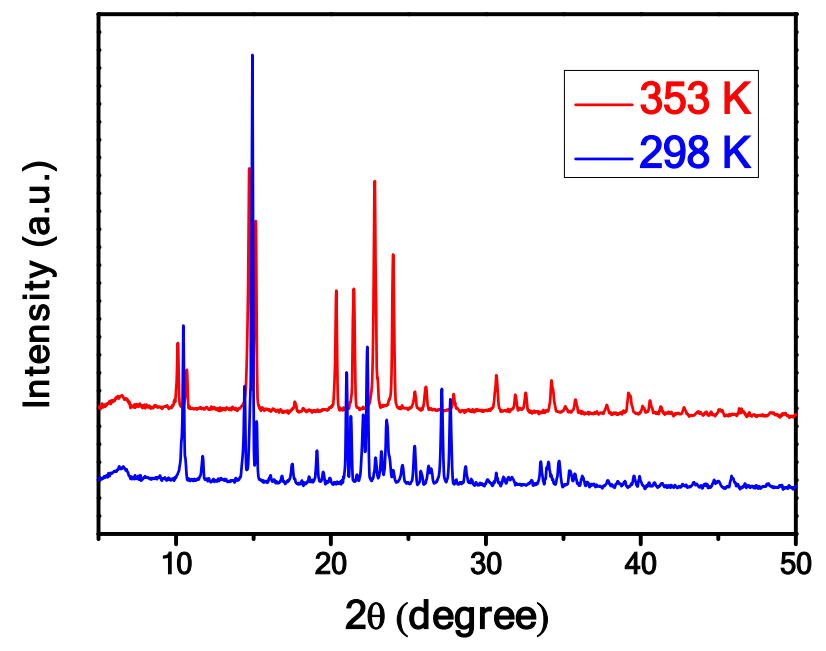

Figure S6. Variable temperature PXRD pattern of 2 measured in the heating process at $298 \mathrm{~K}$ and $353 \mathrm{~K}$.

As depicted in Figure S6, a fairly large number of diffraction peaks vanished and seven new diffraction peaks were discovered at $20.32^{\circ}, 21.48^{\circ}, 22.82^{\circ}, 24.02^{\circ}, 31.90^{\circ}, 32.56^{\circ}$ and $39.34^{\circ}$. At the same time, the diffraction peaks at $10.14^{\circ}, 14.76^{\circ}, 26.08^{\circ}, 34.32^{\circ}$ displayed some shifts. In summary, the varieties of diffraction peaks provide the strong evidence of the occurrence of the phase transition. According to using the Material Studio software, simulating the cell parameters of $\mathbf{2}$ indicates that it crystallizes in tetragonal space group $I 4 / \mathrm{mcm}$.
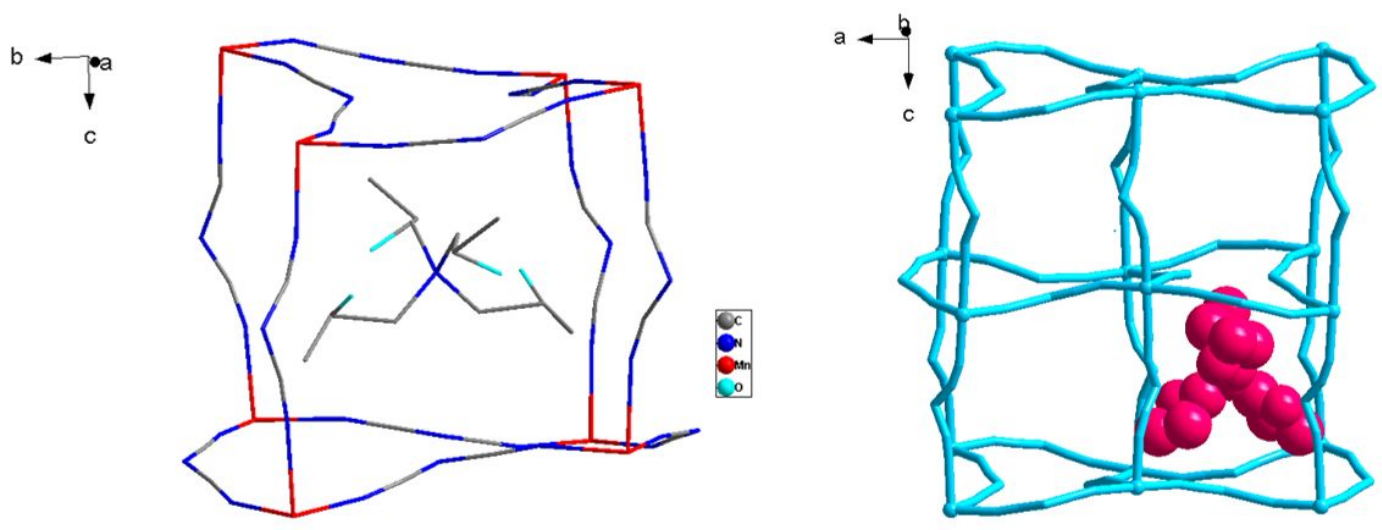

(a) 

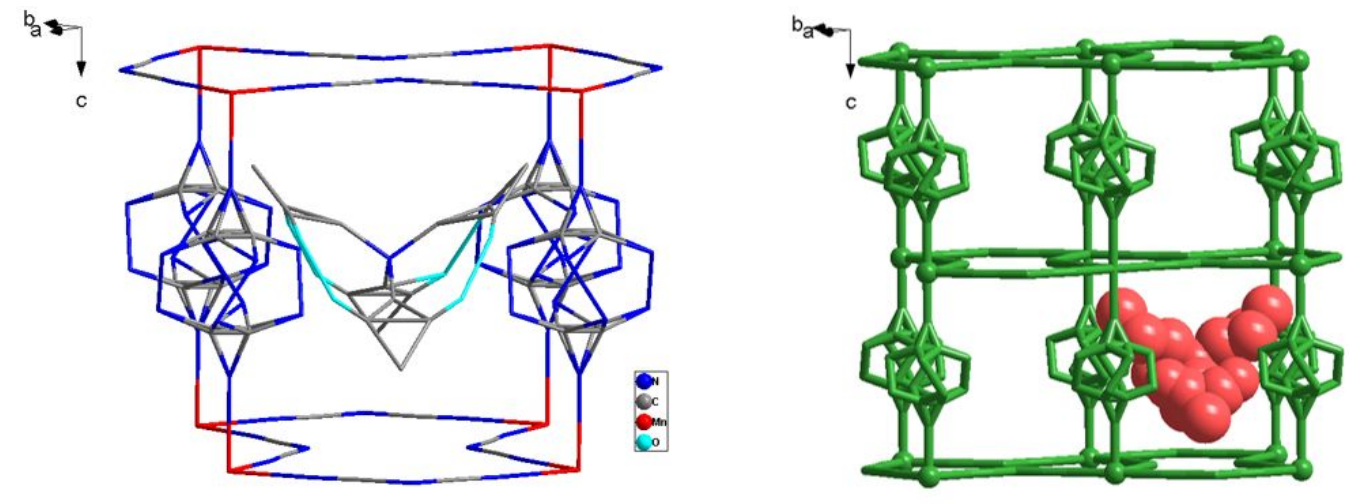

(b)
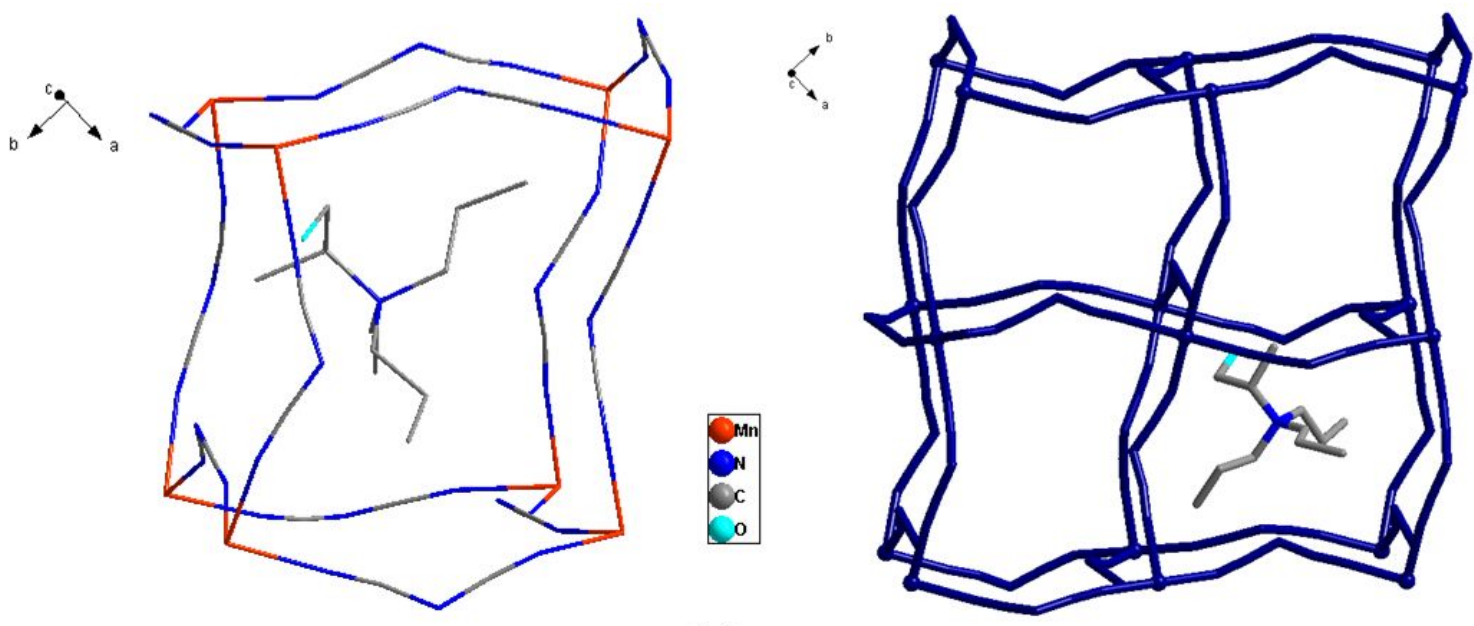

(c)
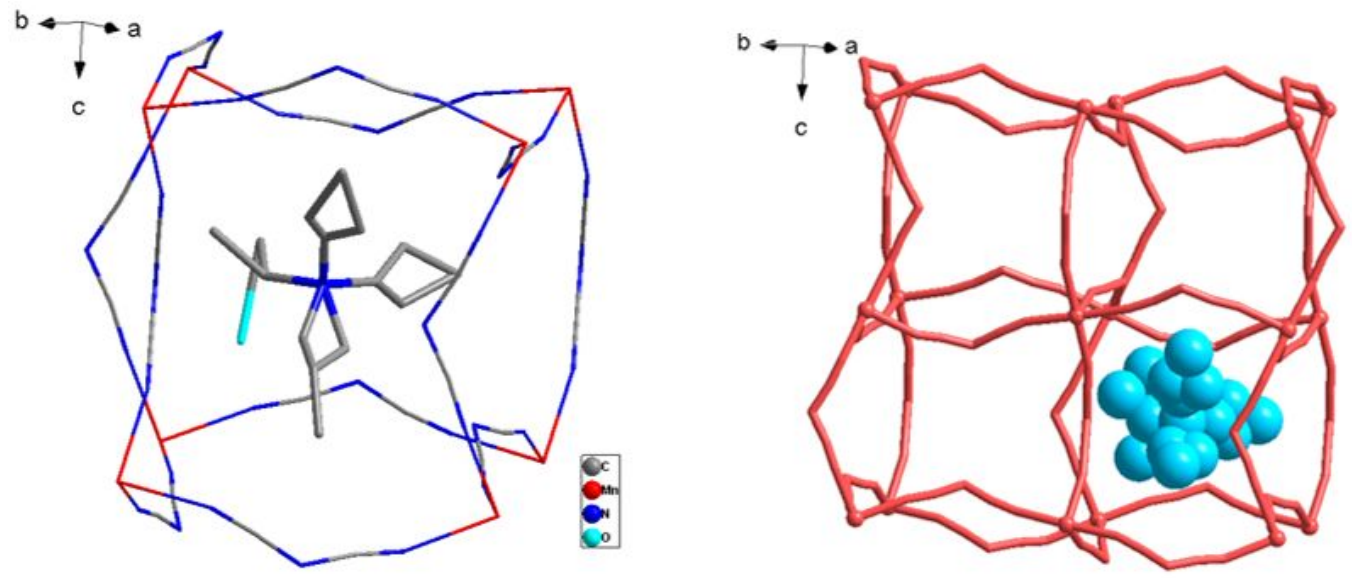

(d) 

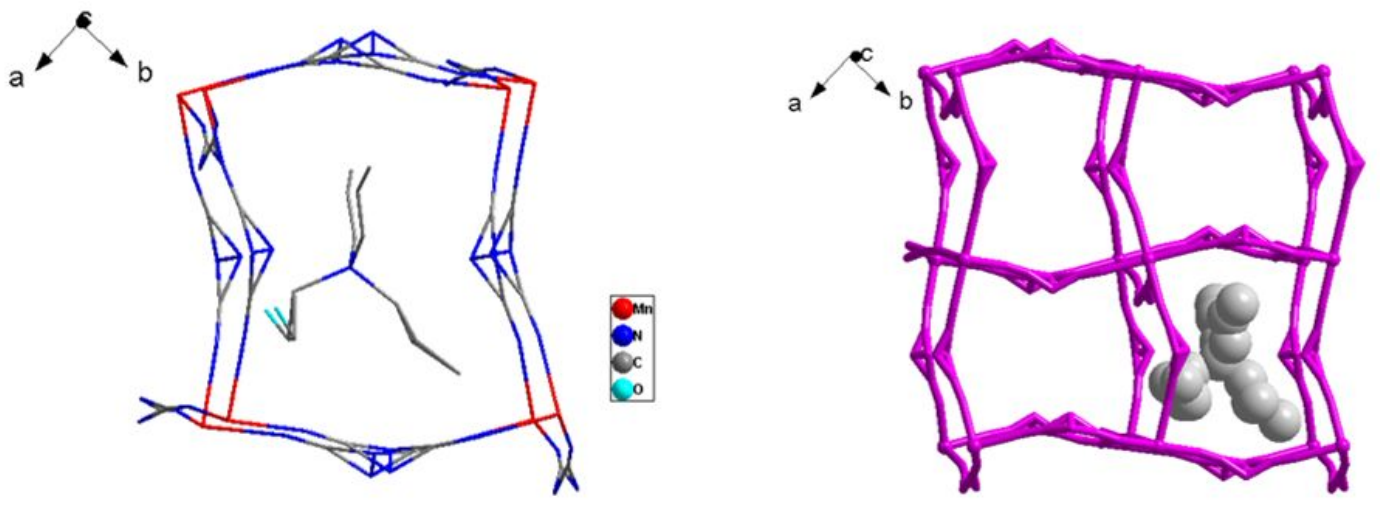

(e)

Figure S7. Crystal structures of 1 in (a) low temperature phase (LTP) and (b) high temperature phase (HTP) and $\mathbf{2}$ in (c) I phase, (d) II phase and (e) III phase. The H atoms of the cations have been removed to facilitate visualization of the structure.

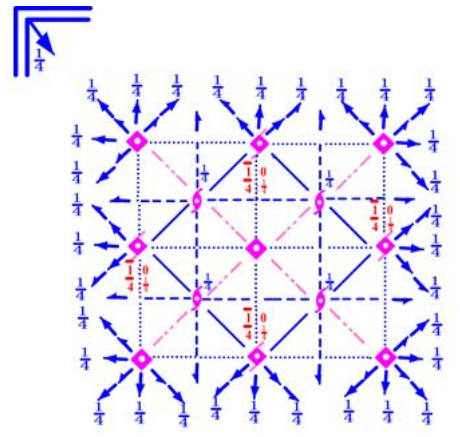

Space group $I 4 / \mathrm{mcm}$ (No.140)

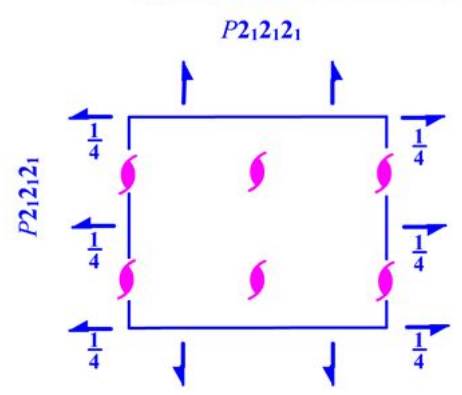

Space group $P 212121$ (No.19) (a)

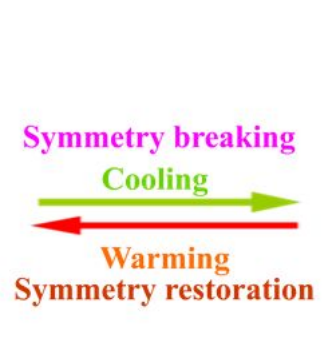

(b)

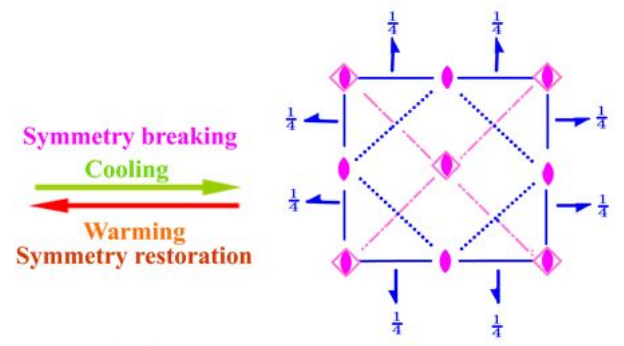

Space group $\bar{P} \overline{4} 21 c($ No.114)

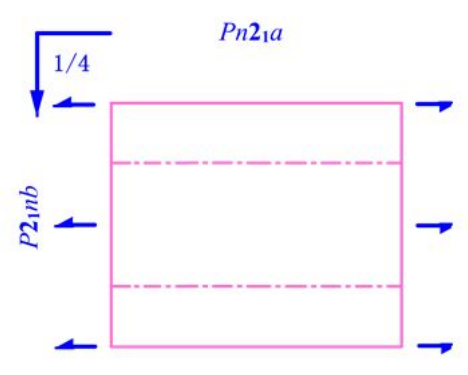

Space group Pna21 (No.33) 


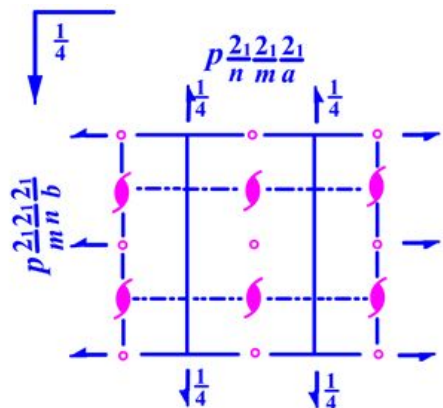

Space group Pnam (No.62)
Symmetry breaking

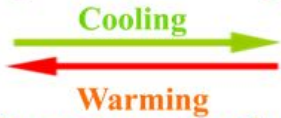

Symmetry restoration

(c)

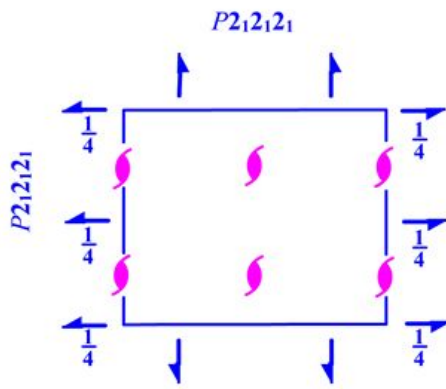

Space group $P 212121($ No.19)

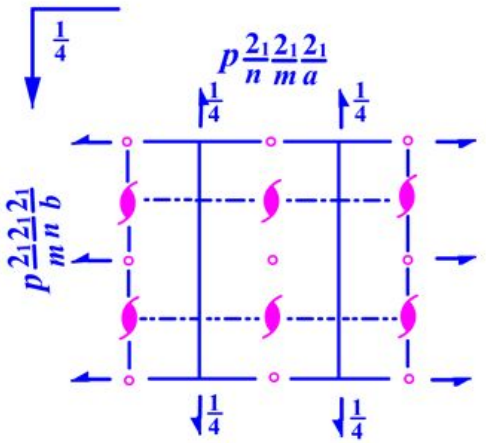

Space group Pnam (No.62)

Space group $I 4 / \mathrm{mcm}$ (No.140)

(d)

Symmetry breaking Cooling

Warming

Symmetry restoration

(a) from LTP to HTP, 2 (b) from I

Figure S8. Symmetry transformations of space groups of $\mathbf{1}$ (a) from LTP to HTP,
phase to II phase, (c) from II phase to III phase and (d) from III phase to IV phase.

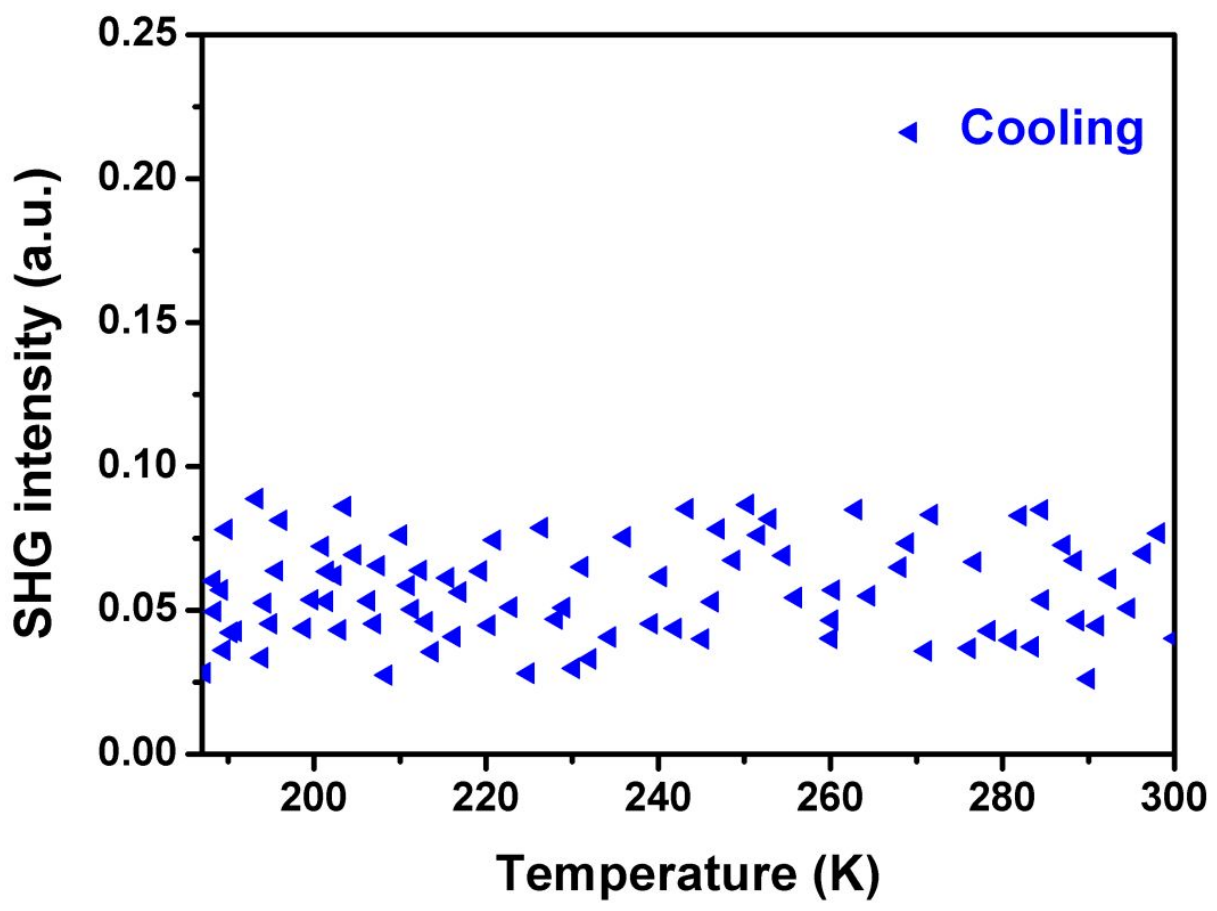

Figure S9. The SHG signals of the compound 1. 


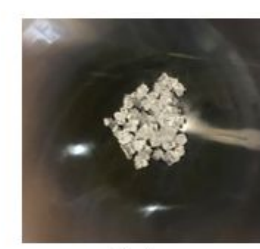

(a)

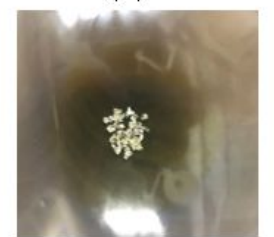

(c)

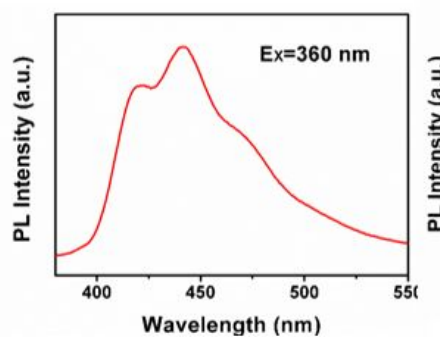

(e)

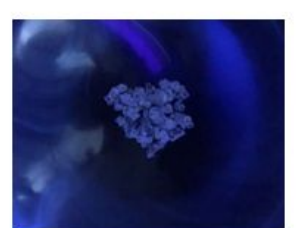

(b)

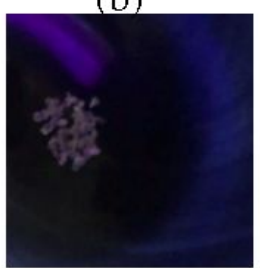

(d)

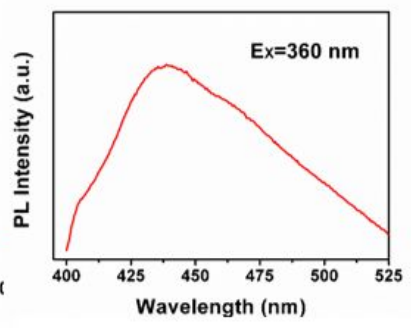

(f)

Figure S10. The crystal photos of 1 under ambient (a) and under UV light (b), and 2 under ambient (c) and under UV light (d). The emission spectra of powder samples for $\mathbf{1}$ (e) and $\mathbf{2}$ (f). According to the above results, there is no obvious difference between different conditions. Luminescence properties are invisible to the naked eyes under the 365 / $254 \mathrm{~nm}$ UV lamp irradiation. And the signals are relatively weak in the photoluminescence spectra.

Table S1. Crystal data, data collection and refinement parameters of crystals of $\mathbf{1}$ and $\mathbf{2}$.

\begin{tabular}{|c|c|c|c|c|c|}
\hline & $143 \mathrm{~K}$ & $293 \mathrm{~K}$ & $193 \mathrm{~K}$ & $282 \mathrm{~K}$ & $301 \mathrm{~K}$ \\
\hline Chemical Formula & \multicolumn{2}{|c|}{$\left[2-\mathrm{OH}-\mathrm{C}_{3} \mathrm{H}_{6}-\mathrm{N}\left(\mathrm{C}_{3} \mathrm{H}_{7}\right)_{3}\right]\left[\mathrm{Mn}(\mathrm{dca})_{3}\right]$} & \multicolumn{3}{|c|}{$\left[2-\mathrm{OH}-1-\mathrm{CH}_{3}-\mathrm{C}_{2} \mathrm{H}_{3}-\mathrm{N}\left(\mathrm{C}_{3} \mathrm{H}_{7}\right)_{3}\right]\left[\mathrm{Mn}(\mathrm{dca})_{3}\right]$} \\
\hline Formula weight & \multicolumn{2}{|c|}{455.38} & \multicolumn{3}{|c|}{455.38} \\
\hline Crystal system & Tetragonal & Tetragonal & Orthorhombic & Orthorhombic & Orthorhombic \\
\hline Space group & $P \overline{4} 2_{1} c$ & $I 4 / \mathrm{mcm}$ & $P n a 2_{1}$ & $P 2_{1} 2_{1} 2_{1}$ & Pnam \\
\hline $\mathrm{a}, \AA$ & $16.1196(3)$ & $11.5389(5)$ & $11.1875(6)$ & $11.6357(6)$ & $11.6485(6)$ \\
\hline $\mathrm{b}, \AA$ & $16.1196(3)$ & $11.5389(5)$ & $12.4566(6)$ & $12.2736(6)$ & $12.2688(5)$ \\
\hline $\mathrm{c}, \AA$ & $17.5219(6)$ & $17.7377(16)$ & $16.4477(8)$ & $16.6608(7)$ & $16.6697(7)$ \\
\hline$\alpha, \operatorname{deg}$ & 90.00 & 90.00 & 90.00 & 90.00 & 90.00 \\
\hline$\beta, \operatorname{deg}$ & 90.00 & 90.00 & 90.00 & 90.00 & 90.00 \\
\hline$\gamma, \operatorname{deg}$ & 90.00 & 90.00 & 90.00 & 90.00 & 90.00 \\
\hline$V, \AA^{3}$ & $4552.9(2)$ & $2361.7(3)$ & $2292.1(2)$ & $2379.4(2)$ & $2382.32(19)$ \\
\hline Z & 4 & 2 & 4 & 4 & 4 \\
\hline$F(000)$ & 1928 & 1064 & 844 & 848 & 844 \\
\hline$\mu, \mathrm{mm}^{-1}$ & 0.62 & 0.61 & 0.60 & 0.58 & 0.58 \\
\hline Radiation (Mo K $\alpha$ ) & 0.71073 & 0.71073 & 0.71073 & 0.71073 & 0.71073 \\
\hline Reflections measured & 18879 & 3723 & 11079 & 12559 & 10949 \\
\hline
\end{tabular}




\begin{tabular}{lccccc} 
Reflections independent & 3990 & 583 & 3673 & 4128 & 2058 \\
Reflections used & 7661 & 1463 & 7590 & 5647 & 1845 \\
Goodness-of-fit on $\mathrm{F}^{2}$ & 1.01 & 1.10 & 1.14 & 1.09 & 1.10 \\
Final $R$ indices & $R_{1}=0.126$ & $R_{1}=0.130$ & $R_{1}=0.103$ & $R_{1}=0.062$ & $R_{1}=0.137$ \\
{$[(I>$ 2sigama $(I))$} & $w R_{2}=0.284$ & $w R_{2}=0.272$ & $w R_{2}=0.304$ & $w R_{2}=0.194$ & $w R_{2}=0.304$ \\
\hline
\end{tabular}

For crystal 2 at 193 K, we have tried to collect better quality data. However, due to the ordered-disordered type of structural phase transition, the bond length and bond angle in the structure twist and stretch strongly. As a result, the diffraction point becomes significantly weaker at low temperature. It matches with the viewpoint of Yaghi O. M et al. ${ }^{1}$ The diffraction spot in the electron cloud diffraction pattern dispersed relatively. When temperature increases to room temperature from low temperature, the electron cloud diffraction will recover and data quality become relatively high. This is a reversible phase transition. Moreover, as for most structural phase transitions, the diffraction quality of the structure after phase transition will decrease.

Table S2. The key bond distances and angles of 1 at $143 \mathrm{~K}$ and $293 \mathrm{~K}$.

\begin{tabular}{|c|c|c|c|c|}
\hline \multirow{8}{*}{$143 \mathrm{~K}$} & Mn1-N1 & $2.213(3)$ & Mn1-N2 & $2.217(4)$ \\
\hline & Mn1-N3 & $2.238(4)$ & Mn1-N4 & $2.244(5)$ \\
\hline & Mn1-N5 & $2.202(4)$ & Mn1-N6 & $2.202(5)$ \\
\hline & $\mathrm{C} 1-\mathrm{N} 7-\mathrm{C} 3^{\mathrm{i}}$ & $110.9(8)$ & $\mathrm{C} 2-\mathrm{N} 8-\mathrm{C} 4{ }^{\mathrm{iii}}$ & $127.3(5)$ \\
\hline & $\mathrm{C} 5^{\text {iv }}-\mathrm{N} 9-\mathrm{C} 6$ & $123.2(5)$ & $\mathrm{C} 1-\mathrm{N} 1-\mathrm{Mn} 1$ & $163.3(5)$ \\
\hline & $\mathrm{C} 5-\mathrm{N} 2-\mathrm{Mn} 1$ & $170.5(4)$ & $\mathrm{C} 6-\mathrm{N} 3-\mathrm{Mn} 1$ & $162.0(5)$ \\
\hline & $\mathrm{C} 2-\mathrm{N} 4-\mathrm{Mn} 1$ & $159.4(5)$ & $\mathrm{C} 3-\mathrm{N} 5-\mathrm{Mn} 1$ & $175.7(8)$ \\
\hline & $\mathrm{C} 4-\mathrm{N} 6-\mathrm{Mn} 1$ & $172.9(5)$ & & \\
\hline
\end{tabular}

Symmetry codes: (i) $\mathrm{y}+1 / 2, \mathrm{x}-1 / 2, \mathrm{z}-1 / 2$; (ii) $-\mathrm{x}+1,-\mathrm{y}+2$, $\mathrm{z}$; (iii) $\mathrm{x}+1 / 2,-\mathrm{y}+1 / 2,-\mathrm{z}+1 / 2$; (iv) $-\mathrm{x}+3 / 2, \mathrm{y}+1 / 2,-\mathrm{z}+1 / 2 ;$ (v) $-\mathrm{x}+3 / 2, \mathrm{y}-1 / 2,-\mathrm{z}+1 / 2$; (vi) $\mathrm{x}-1 / 2,-\mathrm{y}+1 / 2,-\mathrm{z}+1 / 2$; (vii) $\mathrm{y}+1 / 2$, $\mathrm{x}-1 / 2, \mathrm{z}+1 / 2$; (viii) $\mathrm{y}+1,-\mathrm{x}+1,-\mathrm{z}$; (ix) $-\mathrm{x}+2,-\mathrm{y}, \mathrm{z}$; (x) $-\mathrm{y}+1, \mathrm{x}-1,-\mathrm{z}$; (xi) $\mathrm{y},-\mathrm{x}+1,-\mathrm{z}$; (xii) $-\mathrm{x}+1,-\mathrm{y}+1, \mathrm{z} ;(\mathrm{xiii})-\mathrm{y}+1, \mathrm{x},-\mathrm{z}$.

\begin{tabular}{|l|l|l|l|l|}
\hline \multirow{4}{*}{$293 \mathrm{~K}$} & $\mathrm{Mn} 1-\mathrm{N} 1$ & $2.226(5)$ & Mn1-N1 & $2.226(5)$ \\
\cline { 2 - 5 } & $\mathrm{Mn} 1-\mathrm{N} 1^{\mathrm{ii}}$ & $2.226(5)$ & $\mathrm{Mn} 1-\mathrm{N} 1^{\mathrm{iii}}$ & $2.226(5)$ \\
\cline { 2 - 5 } & $\mathrm{Mn} 1-\mathrm{N} 3$ & $2.065(6)$ & $\mathrm{Mn} 1-\mathrm{N} 3^{\mathrm{i}}$ & $2.065(6)$ \\
\cline { 2 - 5 } & & & & \\
\cline { 2 - 5 } & $\mathrm{C} 1^{\mathrm{iv}}-\mathrm{N} 2-\mathrm{C} 1$ & $123.4(7)$ & $\mathrm{C} 1-\mathrm{N} 1-\mathrm{Mn} 1$ & $170.0(4)$ \\
\hline
\end{tabular}

Symmetry codes: (i) $-\mathrm{x},-\mathrm{y},-\mathrm{z}$; (ii) $\mathrm{y},-\mathrm{x},-\mathrm{z}$; (iii) $-\mathrm{y}, \mathrm{x}, \mathrm{z}$; (iv) $-\mathrm{y}+1 / 2,-\mathrm{x}+1 / 2, \mathrm{z}$; (v) $-\mathrm{x}+1$, $-\mathrm{y}+2, \mathrm{z}$; (vi) $-\mathrm{y}+3 / 2, \mathrm{x}+1 / 2,-\mathrm{z}+1 / 2$; (vii) $\mathrm{y}-1 / 2,-\mathrm{x}+3 / 2,-\mathrm{z}+1 / 2$; (viii) $\mathrm{y}-1 / 2, \mathrm{x}+1 / 2, \mathrm{z}$.

Table S2. The key bond distances and angles of 2 at $193 \mathrm{~K}, 282 \mathrm{~K}$ and $293 \mathrm{~K}$.

\begin{tabular}{|l|l|l|l|l|}
\hline & $\mathrm{Mn} 1-\mathrm{N} 1$ & $2.213(5)$ & $\mathrm{Mn} 1-\mathrm{N} 2$ & $2.190(5)$ \\
\cline { 2 - 6 } & $\mathrm{Mn} 1-\mathrm{N} 3$ & $2.229(4)$ & $\mathrm{Mn} 1-\mathrm{N} 4$ & $2.209(5)$ \\
\cline { 2 - 5 } & $\mathrm{Mn} 1-\mathrm{N} 5$ & $2.210(5)$ & Mn1-N6 & $2.243(5)$ \\
\hline
\end{tabular}




\begin{tabular}{|l|l|l|l|l|}
\hline $193 \mathrm{~K}$ & & & & \\
\cline { 2 - 5 } & $\mathrm{C} 5-\mathrm{N} 7-\mathrm{C} 6$ & $123.5(5)$ & $\mathrm{C} 4-\mathrm{N} 8-\mathrm{C} 1^{\mathrm{v}}$ & $121.0(6)$ \\
\cline { 2 - 6 } & $\mathrm{C} 3-\mathrm{N} 9-\mathrm{C} 2^{\mathrm{vi}}$ & $120.7(6)$ & $\mathrm{C} 2-\mathrm{N} 1-\mathrm{Mn} 1$ & $165.0(5)$ \\
\cline { 2 - 6 } & $\mathrm{C} 1-\mathrm{N} 2-\mathrm{Mn} 1$ & $157.1(5)$ & $\mathrm{C} 6-\mathrm{N} 3-\mathrm{Mn} 1$ & $166.1(4)$ \\
\hline & $\mathrm{C} 3-\mathrm{N} 4-\mathrm{Mn} 1$ & $157.7(5)$ & $\mathrm{C} 4-\mathrm{N} 5-\mathrm{Mn} 1$ & $164.7(5)$ \\
\hline & $\mathrm{C} 5-\mathrm{N} 6-\mathrm{Mn} 1$ & $147.8(4)$ & & \\
\hline
\end{tabular}

Symmetry codes: (i) $\mathrm{x}+1 / 2,-\mathrm{y}+3 / 2$, $\mathrm{z}$; (ii) $-\mathrm{x},-\mathrm{y}+1, \mathrm{z}-1 / 2$; (iii) $\mathrm{x}-1 / 2,-\mathrm{y}+1 / 2$, $\mathrm{z}$; (iv) $\mathrm{x}-1 / 2$, $-\mathrm{y}+3 / 2, \mathrm{z} ;(\mathrm{v})-\mathrm{x},-\mathrm{y}+1, \mathrm{z}+1 / 2 ;$ (vi) $\mathrm{x}+1 / 2,-\mathrm{y}+1 / 2, \mathrm{z}$.

\begin{tabular}{|c|c|c|c|c|}
\hline \multirow{8}{*}{$282 \mathrm{~K}$} & Mn1-N1 & $2.2064(17)$ & Mn1-N2 & $2.2164(14)$ \\
\hline & Mn1-N3 & $2.2133(17)$ & Mn1-N4 & $2.2162(14)$ \\
\hline & Mn1-N5 & $2.2275(17)$ & Mn1-N6 & $2.2009(16)$ \\
\hline & $\mathrm{C} 5^{\mathrm{v}}-\mathrm{N} 7-\mathrm{C} 1$ & $123.3(2)$ & $\mathrm{C} 2-\mathrm{N} 8-\mathrm{C} 4^{\mathrm{ii}}$ & $120.43(19)$ \\
\hline & $\mathrm{C} 3-\mathrm{N} 9-\mathrm{C}^{\mathrm{vi}}$ & $124.0(2)$ & $\mathrm{C} 1-\mathrm{N} 1-\mathrm{Mn} 1$ & $163.94(18)$ \\
\hline & $\mathrm{C} 2-\mathrm{N} 2-\mathrm{Mn} 1$ & $165.95(16)$ & $\mathrm{C} 3-\mathrm{N} 3-\mathrm{Mn} 1$ & $170.39(17)$ \\
\hline & $\mathrm{C} 4-\mathrm{N} 4-\mathrm{Mn} 1$ & $166.70(16)$ & $\mathrm{C} 5-\mathrm{N} 5-\mathrm{Mn} 1$ & $158.39(17)$ \\
\hline & C6-N6-Mn1 & $166.74(17)$ & & \\
\hline
\end{tabular}

Symmetry codes: (i) $-\mathrm{x}+3 / 2,-\mathrm{y}+1, \mathrm{z}-1 / 2$; (ii) $-\mathrm{x}+3 / 2,-\mathrm{y}+1, \mathrm{z}+1 / 2$; (iii) $-\mathrm{x}+2, \mathrm{y}+1 / 2,-\mathrm{z}+1 / 2$; (iv) $-\mathrm{x}+1, \mathrm{y}-1 / 2,-\mathrm{z}+1 / 2 ;$ (v) $-\mathrm{x}+2, \mathrm{y}-1 / 2,-\mathrm{z}+1 / 2$; (vi) $-\mathrm{x}+1, \mathrm{y}+1 / 2,-\mathrm{z}+1 / 2$.

\begin{tabular}{|l|l|l|l|l|}
\hline \multirow{5}{*}{$301 \mathrm{~K}$} & $\mathrm{Mn} 1-\mathrm{N} 1$ & $2.217(3)$ & $\mathrm{Mn} 1-\mathrm{N} 1^{\mathrm{i}}$ & $2.217(3)$ \\
\cline { 2 - 5 } & $\mathrm{Mn} 1-\mathrm{N} 7$ & $2.209(3)$ & $\mathrm{Mn} 1-\mathrm{N} 7^{\mathrm{i}}$ & $2.209(3)$ \\
\cline { 2 - 5 } & $\mathrm{Mn} 1-\mathrm{N} 4^{\mathrm{ii}}$ & $2.218(3)$ & $\mathrm{Mn} 1-\mathrm{N} 4^{\mathrm{iii}}$ & $2.218(3)$ \\
\cline { 2 - 5 } & & & & \\
\cline { 2 - 5 } & $\mathrm{C} 3-\mathrm{N} 2-\mathrm{C} 5$ & $117.4(5)$ & $\mathrm{C} 5-\mathrm{N} 2^{\prime}-\mathrm{C} 3$ & $115.7(5)$ \\
\cline { 2 - 5 } & $\mathrm{C} 1-\mathrm{N} 9-\mathrm{C} 1^{\mathrm{v}}$ & $119.1(6)$ & $\mathrm{C} 1-\mathrm{N} 9{ }^{\prime}-\mathrm{C} 1^{\mathrm{v}}$ & $113.9(7)$ \\
\hline & $\mathrm{C} 5-\mathrm{N} 1-\mathrm{Mn} 1$ & $163.0(3)$ & $\mathrm{C} 3-\mathrm{N} 4-\mathrm{Mn} 1^{\mathrm{iv}}$ & $167.3(4)$ \\
\hline & $\mathrm{C} 1-\mathrm{N} 7-\mathrm{Mn} 1$ & $167.9(3)$ & & \\
\hline
\end{tabular}

Symmetry codes: (i) $-\mathrm{x}+1,-\mathrm{y}+1,-\mathrm{z}$; (ii) $-\mathrm{x}+1 / 2, \mathrm{y}-1 / 2,-\mathrm{z}$; (iii) $\mathrm{x}+1 / 2,-\mathrm{y}+3 / 2, \mathrm{z}$; (iv) $-\mathrm{x}+1 / 2$, $\mathrm{y}+1 / 2,-\mathrm{z}$; (v) $\mathrm{x}, \mathrm{y},-\mathrm{z}+1 / 2$; (vi) $\mathrm{x}, \mathrm{y},-\mathrm{z}+3 / 2$.

1. Lee, S.; Burgi, H. B.; Alshmimri, S. A.; Yaghi, O. M. Impact of Disordered Guest-Framework Interactions on the Crystallography of Metal-Organic Frameworks. J. Am. Chem. Soc. 2018, 140 (28), 8958-8964. 